\title{
The hydraulic resistance in the small-scale pillow-plate heat exchangers
}

\section{Olga Arsenyeva}

Department of integrated technologies processes and apparatuses, National technical university "Kharkiv polytechnic institute", Ukraine, Kharkiv, 2 Kyrpichova Street, E-mail: o.arsenyeva@cfe.org.ua

The Pillow-Plate Heat Exchangers (PPHEs) are rated as innovative type of heat transfer equipment. The present paper gives the information about the research made in Paderborn University aimed to investigate the heat transfer and pressure drop in the channels of the small-scale PPHE.

Keywords - heat exchangers, pillow plates, heat transfer, heat exchanger design, hydraulic resistance.

\section{Abstract}

The so-called Pillow-Plate Heat Exchangers (PPHEs) are innovative type of heat exchange equipment. They have space-effective, light and pressure-resistant construction and show intensified heat transfer and low pressure loss on the product media side [1]. PPHEs are produced by spot-welding of two steel sheets, followed by hydro-forming to obtain channels for heat carrier movement. In case of condensers, the cooling medium flows on the inner side forming a cooling channel, whereas the vapour flows on the outer side (between cooling channels). The edges are fully seam-welded. The schematic of a pillow plate with its main geometrical parameters are presented in Fig. 1. Pillow-plate surfaces of PPHEs ensure the turbulent movement of heat carriers inside the channels formed by the plates. The particular waviness of the pillow-plate channels promotes lateral mixing and beneficial turbulence, which results in a good thermo-hydraulic performance. The manufacturing technology of PPHEs is extremely flexible, and the diversity of the resulting geometries is immense. The application area of PPHEs is broad and expanding. In contrast to conventional equipment, however, no reliable design methods for compact PPHEs are available in the open literature [2].

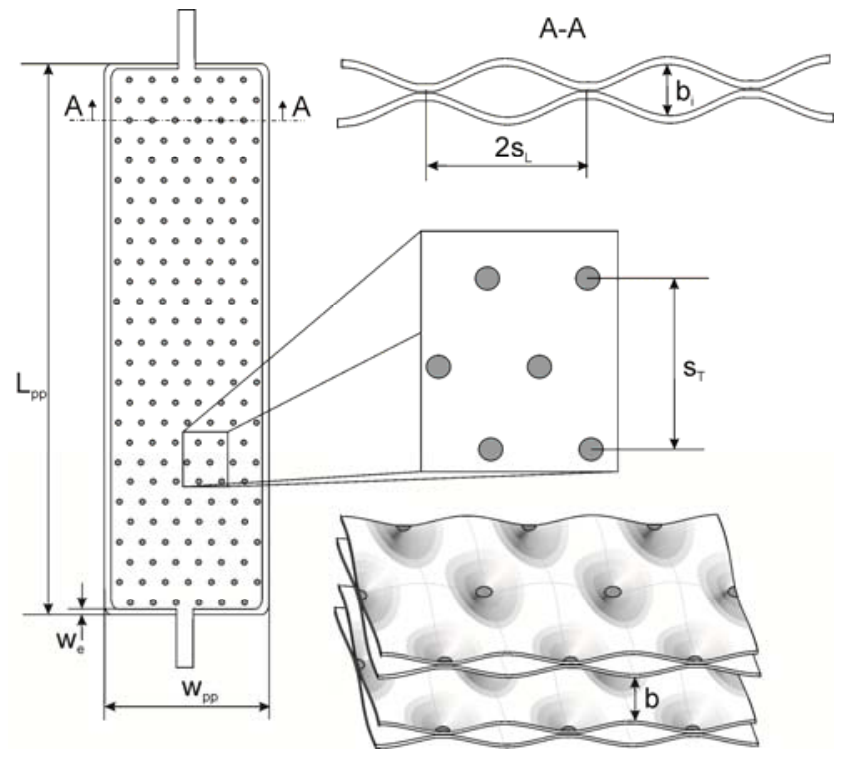

Fig.1. The schematic of pillow plate with specification of its main geometric parameters.

The reliable design of PPHEs includes the identification of the geometrical parameters (gap width, height and length of the plates) and estimation of heat transfer and hydraulic parameters of the unit and is mostly influenced by the geometry of the heat transfer channels, 
which determine the heat transfer and hydraulic parameters of the unit. The standard pillow plates are assembled together in one unit, what enables to vary the distance between them, and consequently adjust the hydraulic resistance and heat transfer process in the outer channels between welded pillow plates (external or E-channel). The geometry of inner-plate channel (inner or I-channel) determines the whole performance of the PPHE. The geometries of Ichannels for the investigated small-scale PPHE are presented in [3]. The description of the experimental set-up for investigation of hydraulic resistance in small-scale PPHE is published in [3]. The empirical equations for calculation of friction factors in the I-channel, which has smaller cross-section area, $\zeta_{1}$ was derived from the experimental data based on the form of equation proposed by Churchill [4] for straight tubes and has the following form:

$$
\zeta=8 \cdot\left[\left(\frac{12+p 2}{\operatorname{Re}}\right)^{12}+\frac{1}{(A+B)^{\frac{3}{2}}}\right]^{\frac{1}{12}} ; A=\left[p 4 \cdot \ln \left(\frac{p 5}{\left(\frac{7 \cdot p 3}{R e}\right)^{0.9}+0.27 \cdot 10^{-5}}\right)\right]^{6} ; B=\left(\frac{37530 \cdot p 1}{R e}\right)^{16}
$$

where $p 1$ to $p 5$ are parameters defined by the channel form and for the investigated smallscale PPHE are equal: $p 1=136.321 ; p 2=7.387 ; p 3=0.382 ; p 4=0.515 ; p 5=4.622$.

The friction factor in E-channel $\zeta_{2}$ was obtained based on the experimental data, and has the following form:

$$
\zeta=0.7155 \cdot R e^{-0.361}
$$

Values calculated according to Eqs. (1), (2) differ from experimental data by less than $\pm 10 \%$ and are valid in the range of Reynolds numbers between 2,000 and 20,000.

\section{Conclusion}

In this work, the experimental data for hydraulic resistance in small-scale PPHE were obtained. The correlations for friction factor in PPHEs inner and outer channel were worked out, with a deviation from experimental results within $10 \%$. They are reliable for the Reynolds numbers varying between 2000 and 20000.

\section{Acknowledgments}

Olga Arsenyeva is grateful to the Alexander von Humboldt Foundation for the financial support.

\section{References}

[1] J. Tran, S. Sommerfeld, M. Piper, E. Kenig, Investigation of pillow-plate condensers for the application in distillation columns, "Chemical Engineering Research and Design", vol. 99, pp. 67-74, 2015.

[2] M. Piper, C. Wecker, A. Olenberg, J. Tran, E. Kenig, An experimental analysis of the topology and dynamics of a falling liquid film over the wavy surface of a vertical pillow plate, "Chemical Engineering Science", vol. 130, pp. 129-134, 2015.

[3] O.P. Arsenyeva, M. Piper, A. Zibart, A. Olenberg, E. Kenig, Heat transfer and pressure loss in small-scale pillow-plate heat exchangers, "Chemical Engineering Transactions", vol. 70, pp. 799-804, 2018.

[4] S.W. Churchill, Friction factor equation spans all fluid flow regimes, "Chemical Engineering”, vol. 84, pp. 91-92, 1977. 